\title{
Zur geometrischen Erzeugung linearer Geradenabbildungen
}

\author{
Von \\ Boris Odehnal \\ (Vorgelegt in der Sitzung der math.-nat. Klasse am 24. Juni 2004 \\ durch das k. M. Hellmuth Stachel)
}

\section{Zusammenfassung}

In der vorliegenden Arbeit wird gezeigt, daß die surjektiven linearen Geradenabbildungen aus der Mannigfaltigkeit $\mathcal{L}$ der Geraden des projektiven Dreiraumes $\mathbb{P}^{3}$ in den $\mathbb{P}^{3}$ bis auf eine Ausnahme durch die Zusammensetzung einer Netzprojektion, einer stereographischen Projektion und einer anschließenden Polarität im $\mathbb{P}^{3}$ realisiert werden können. Ferner werden die geometrische Realisierung der linearen Geradenabbildungen $\mathcal{L} \rightarrow \mathbb{P}^{2}$ diskutiert und die Zusammenhänge zwischen den linearen Geradenabbildungen in $\mathbb{P}^{3}$ und in $\mathbb{P}^{2}$ untersucht.

Mathematics Subject Classification (2000): 51M15, 51M30, 51N15.

Key words: Line geometry, linear mapping, linear line mapping, stereographic projection, quadric, regulus, conic.

\section{Einleitung}

Lineare Geradenabbildungen wurden erstmalig von L. ECKHART in $[7,8]$ und F. REHBOCK in $[18,19]$ untersucht. Eine Geradenabbildung wurde dann als linear bezeichnet, wenn ihre Abbildungsgleichungen linear in den PLÜCKER-Koordinaten sind. Beide Autoren beschäftigen sich eingehend mit der Abbildung von Geraden des drei-dimensionalen projektiven Raumes $\mathbb{P}^{3}$ auf Punkte einer projektiven Ebene $\mathbb{P}^{2}$. Neben der synthetischen Behandlung der linearen Abbildungsverfahren werden auch die Verbindungen der linearen Geradenabbildungen zur Kinematik aufgezeigt. 
Auch die kinematische Abbildung von BLASCHKE [2] und GRÜNWALD [10], welche die Geraden des drei-dimensionalen quasi-elliptischen Raumes auf Punktepaare einer euklidischen Ebene abbildet, ist eine lineare Geradenabbildung. Die zum Studium des Geradenkontinuums des drei-dimensionalen elliptischen Raumes $\mathbb{E}^{3}$ vorteilhafte sphärische kinematische Abbildung von W. K. CLIFFORD [5] - siehe aber auch $[3,15,17]$ - ist ebenfalls zu den linearen Geradenabbildungen $\mathrm{zu}$ zählen.

Grundlage der folgenden Überlegungen ist zum einen die nach F. KLEIN benannte Abbildung $\gamma: \mathcal{L} \rightarrow M_{2}^{4}$, welche den Geraden des $\mathbb{P}^{3}$ die Punkte der PLÜCKER-Quadrik $M_{2}^{4} \subset \mathbb{P}^{5}$ zuweist [9, 17, 21], und zum anderen die Auffassung einer linearen Abbildung $\lambda: \mathbb{P}^{m} \rightarrow \mathbb{P}^{n}$ $(n<m)$ als Produkt einer Projektion (singulären Kollineation) und einer anschließenden kollinearen Verlagerung des Bildraumes.

H. BRAUNER hat in [4] die folgende auch hier verwendete Definition linearer Geradenabbildungen gegeben:

Definition 1.1. Eine Abbildung $\mu: \mathcal{L} \rightarrow \mathbb{P}^{d}$ aus der Mannigfaltigkeit $\mathcal{L}$ der Geraden des drei-dimensionalen projektiven Raumes $\mathbb{P}^{3}$ heißt lineare Geradenabbildung, wenn sie Zusammensetzung der KLEINschen Abbildung $\gamma$ und einer linearen Abbildung $\lambda: \mathbb{P}^{5} \rightarrow \mathbb{P}^{d}$ ist.

In [4] wurden die linearen Geradenabbildungen in projektive Ebenen $(d=2)$ klassifiziert. Die einzelnen Typen linearer Geradenabbildungen $\mu: \mathcal{L} \rightarrow \mathbb{P}^{2}$ werden hinsichtlich der Lage des Projektionszentrums zur PLÜCKER-Quadrik $M_{2}^{4}$ unterschieden.

Die Arbeit [11] schließt an [4] an und entwickelt Angaben linearer Geradenabbildungen, bei denen die Bilder der Geraden allein durch lineare Konstruktionen ermittelt werden können, was ganz dem Wesen linearer Geradenabbildungen entspricht. Einen analytischen Zugang zum Studium der linearen Geradenabbildungen wählt LÜBBERT in [14]. Eine ausführliche Darstellung linearer Geradenabbildungen, deren synthetische und analytische Behandlung sowie ihre Anwendbarkeit auf die Kinematik der CAYLEY-KLEIN-Räume findet man in [9, 17].

In der vorliegenden Arbeit soll erstmals eine Realisierung der linearen Geradenabbildungen von $\mathcal{L}$ in den $\mathbb{P}^{3}$ ausschließlich innerhalb des $\mathbb{P}^{3}$ vorgenommen werden. Es erweist sich als zielführend, die Projektion aus dem KLEINschen Bild in Konstruktionen in der Geradenmannigfaltigkeit des $\mathbb{P}^{3}$ zu übersetzen.

Ein Teil dieser Realisierung, die Zusammensetzung einer Netzprojektion und die anschließende stereographische Projektion, wurden in [6, 13] zur Konstruktion rationaler Parametrisierungen von Kurven und Flächenstücken in Quadriken benützt. Man sprach 
dort diese zusammengesetzte Abbildung als verallgemeinerte stereographische Projektion an.

\section{Grundlagen aus der Liniengeometrie}

Im folgenden beschreiben wir Punkte $X$ des drei-dimensionalen komplex erweiterten projektiven Raumes $\mathbb{P}^{3}$ durch homogene Koordinaten $\left(x_{0}: x_{1}: x_{2}: x_{3}\right)$. Den Geraden $G$ des $\mathbb{P}^{3}$ weisen wir ihre homogenen PLÜCKER-Koordinaten $\left(g_{1}: g_{2}: g_{3}: g_{4}: g_{5}: g_{6}\right) \mathrm{zu}$, siehe hierzu etwa $[9,12,17,21]$. Für die PLÜCKER-Koordinaten $\left(g_{1}: \ldots: g_{6}\right)$ einer Geraden $G \subset \mathbb{P}^{3}$ gilt dann die PLÜCKER-Identität

$$
M_{2}^{4}: \quad g_{1} g_{4}+g_{2} g_{5}+g_{3} g_{6}=0,
$$

welche gleichzeitig auch die Gleichung der PLÜCKER-Quadrik ist. Die Abbildung $\gamma: \mathcal{L} \rightarrow M_{2}^{4}$, die vermöge $G \mapsto\left(g_{1}: \ldots: g_{6}\right)$ jeder Geraden $G \subset \mathbb{P}^{3}$ (oder $G \in \mathcal{L}$ ) ihre PLÜCKER-Koordinaten zuweist, heißt KLEINsche Abbildung. Die KLEINschen Bilder schneidender Geraden liegen polar bezüglich $M_{2}^{4}$.

Mit Hilfe der KLEINschen Abbildung können die ein-, zwei- oder drei-dimensionalen Geradenmannigfaltigkeiten des $\mathbb{P}^{3}$ einer übersichtlichen Behandlung zugeführt werden, siehe hierzu etwa [17] oder [21].

Man nennt die drei-dimensionale Mannigfaltigkeit der Geraden des $\mathbb{P}^{3}$, deren PLÜCKER-Koordinaten eine lineare Gleichung erfüllen, einen linearen Komplex. Das KLEINsche Bild des linearen Geradenkomplexes ist ein hyperebener Schnitt von $M_{2}^{4}$. Die Geraden, die eine feste Gerade treffen, bilden einen sogenannten singulären linearen Komplex; sein KLEINsches Bild ist der Schnitt von $M_{2}^{4}$ mit einer ihrer Tangentialhyperebenen.

Der Durchschnitt zweier regulärer linearer Komplexe heißt lineare Geradenkongruenz oder Netz. Die PLÜCKER-Koordinaten der Geraden eines Netzes erfüllen zwei lineare Gleichungen. Das KLEINsche Bild der Netzgeraden ist der Schnitt eines drei-dimensionalen projektiven Unterraumes von $\mathbb{P}^{5}$ mit $M_{2}^{4}$, also eine Quadrik in $M_{2}^{4}$.

Man unterscheidet drei Typen von Netzen, je nachdem, ob das KLEINsche Bild eine ovale oder ringartige Quadrik oder ein quadratischer Kegel ist. Erstere heißen elliptische beziehungsweise hyperbolische Netze, letztere heißen parabolische Netze. Das elliptische Netz kann auch als die Menge der Treffgeraden eines Paares konjugiert komplexer windschiefer Geraden $A_{1}$ und $A_{2}$ erzeugt werden. Man nennt $A_{i}$ die Achsen des Netzes. Im hyperbolischen Fall sind diese reell. Das parabolische Netz besteht aus allen Geradenbüscheln, deren 

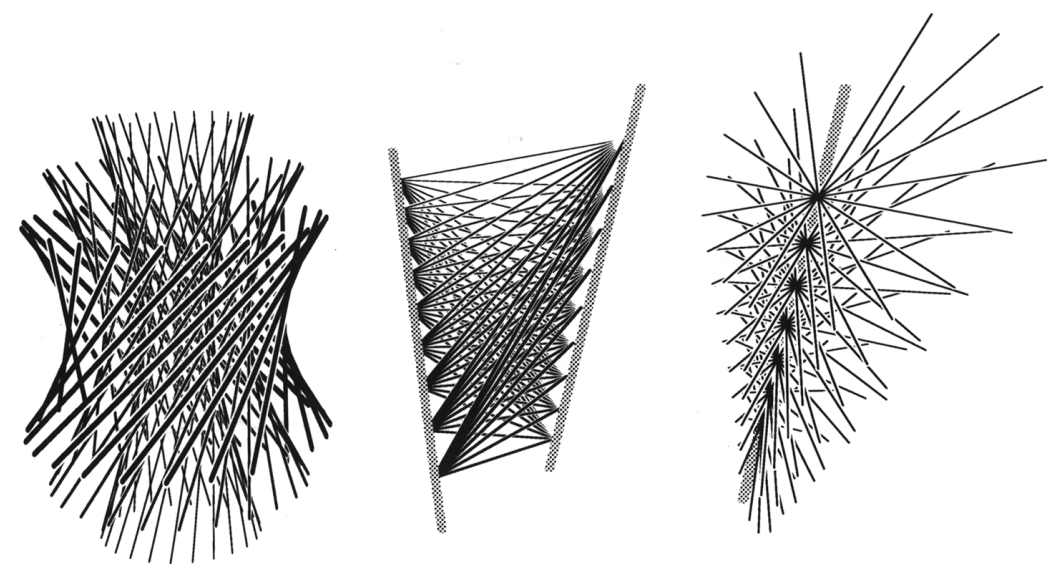

Abb. 1. Von links nach rechts: elliptisches, hyperbolisches und parabolisches Netz

Scheitel auf der einzigen Achse $A$ liegen und deren Ebenen durch $A$ gehen und den Büschelscheiteln in projektiver Weise zugeordnet sind. Nur im parabolischen Fall gehört die Achse zum Netz.

Da nun durch jeden Punkt des $\mathbb{P}^{3}$, der keiner Netzachse angehört, genau eine Netzgerade geht, können Netze zur Abbildung des Punktraumes auf eine Ebene verwendet werden, siehe [1, 7, 8]. $\mathrm{Da}$ wir später davon Gebrauch machen wollen, geben wir die Abbildungsgleichungen für die einzelnen Typen von Netzprojektionen an.

Wir nehmen an, ein elliptisches Netz $\mathcal{N}$ sei durch seine Achsen $A_{1}=(1: i: 0: 1: i: 0)$ und $A_{2}=(1:-i: 0: 1:-i: 0)$ gegeben. Die Projektion $\nu$ erfolge in die Ebene $\mathbb{P}^{2}: x_{3}=0$. Ein Punkt $P \in \mathbb{P}^{3}$ mit den homogenen Koordinaten $\left(p_{0}: p_{1}: p_{2}: p_{3}\right)$ wird dann auf den Punkt

$$
P \nu=\left(p_{0}^{2}+p_{3}^{2}: p_{0} p_{1}+p_{2} p_{3}: p_{0} p_{2}-p_{1} p_{3}: 0\right)
$$

abgebildet. Für den hyperbolischen Fall können wir $A_{1}=$ $(0: 0: 1: 0: 0: 0)$ und $A_{2}=(0: 0: 0: 0: 0: 1)$ annehmen. Hier sei $\mathbb{P}^{2}: x_{0}=x_{1}$ die Bildebene von $\nu$, weshalb ein Punkt $P=$ $\left(p_{0}: p_{1}: p_{2}: p_{3}\right)$ auf den Punkt

$$
P \nu=\left(p_{2} p_{3}: p_{2} p_{3}:-p_{1} p_{3}:-p_{0} p_{2}\right)
$$

abgebildet wird. Im parabolischen Fall wählen wir als Achse $A=(0: 0: 0: 1: 0: 0)$ und, da damit das parabolische Netz noch 
nicht vollständig bestimmt ist, auch noch die Projektivität, die den Punkten von $A$ die Büschelebenen zuordnet. Dazu wählen wir die Nullpolarität $\pi: \mathbb{P}^{3} \rightarrow \mathbb{P}^{3 \star}$, die $A$ festhält und $\pi:\left(p_{0}: p_{1}: p_{2}: p_{3}\right) \mapsto$ $\left(p_{2}:-p_{3}:-p_{1}: p_{0}\right)$ leistet. Ihre Einschränkung auf $A$ liefert genau die gewünschte Abbildung. Als Bildebene der Projektion $\nu$ wählen wir wie im elliptischen Fall die Ebene $\mathbb{P}^{2}: x_{3}=0$ und erhalten als Bild des Punktes $P=\left(p_{0}: p_{1}: p_{2}: p_{3}\right)$ den Punkt

$$
P \nu=\left(p_{0}^{2}:-p_{0} p_{1}:-p_{1} p_{3}+p_{0} p_{2}: 0\right) \text {. }
$$

\section{Die linearen Geradenabbildungen in den $\mathbb{P}^{3}$}

\subsection{Typen linearer Geradenabbildungen in den $\mathbb{P}^{3}$}

Sei $\mathbb{P}^{m}$ ein projektiver Raum der Dimension $m$ und $\mathbb{P}^{n} \subset \mathbb{P}^{m}$ ein projektiver Unterraum von $\mathbb{P}^{m}$ der Dimension $n$. In $\mathbb{P}^{m}$ sei ein $k$-dimensionaler projektiver Unterraum $Z$ ausgezeichnet. Er wird Ausnahmeraum oder Zentrum der linearen Abbildung $\mu$ : $\mathbb{P}^{m} \backslash Z$ $\rightarrow \mathbb{P}^{n}$ in den Bildraum $\mathbb{P}^{n}$ genannt.

Die Ermittlung des Bildpunktes $X \mu \in \mathbb{P}^{n}$ eines Punktes $X \in \mathbb{P}^{m} \backslash Z$ sieht nun folgende Konstruktion vor: Man verbindet $X$ mit $Z$ zum Faserraum $\mathcal{F}_{X}$ von $X$ und ermittelt den Schnitt $X \mu=\mathcal{F}_{X} \cap \mathbb{P}^{n}$. Soll $X \mu$ ein Punkt von $\mathbb{P}^{n}$ sein, so $\mathrm{mu} \operatorname{dim} \mathbb{P}^{n}=n=m-k-1$ gelten, es müssen also Zentrum und Bildraum komplementär sein.

$\mathrm{Um}$ zu linearen Geradenabbildungen $\mu: \mathcal{L} \rightarrow \mathbb{P}^{3}$ im Sinne von Definition $1.1 \mathrm{zu}$ gelangen, muß das Zentrum $Z$ eine Gerade des $\mathbb{P}^{5}$ und der Bildraum ein drei-dimensionaler Unterraum $\mathbb{P}^{3}$ von $\mathbb{P}^{5}$ sein.

Hinsichtlich der Lage von $Z$ zur PLÜCKER-Quadrik unterscheiden wir nun die folgenden vier Typen linearer Geradenabbildungen in den $\mathbb{P}^{3}$ :

1. der elliptische Typ: $Z$ schneidet $M_{2}^{4}$ in einem Paar konjugiert komplexer Punkte,

2. der hyperbolische Typ: $Z$ schneidet $M_{2}^{4}$ in einem Paar reeller Punkte,

3. der parabolische Typ: $Z$ berührt $M_{2}^{4}$ und

4. der singuläre Typ: $Z$ liegt in $M_{2}^{4}$.

Der elliptische, der hyperbolische und der parabolische Typ gestatten eine Konstruktion innerhalb der Geradenmannigfaltigkeit $\mathcal{L}$ des $\mathbb{P}^{3}$. Der singuläre Typ konnte bislang nicht durch Konstruktion im $\mathbb{P}^{3}$ realisiert werden. 


\subsection{Geometrische Erzeugung der linearen Geradenabbildungen}

Zur geometrischen Erzeugung linearer Geradenabbildungen werden die Begriffe aus dem KLEINschen Bild in die Geradenmannigfaltigkeit des $\mathbb{P}^{3}$ übertragen. Dabei entspricht dem geradlinigen Zentrum $Z \subset \mathbb{P}^{5}$ ein Büschel linearer Komplexe im $\mathbb{P}^{3}$, also ein Netz $\mathcal{N}$. Die KLEINschen Bilder der Netzgeraden sind die Punkte des Polarraumes $\widehat{Z}$ von $Z$ bezüglich $M_{2}^{4}$.

Das Ermitteln der Faserebene $\mathcal{F}_{X}$ ist gleichbedeutend mit dem Aufsuchen der ringartigen Faserquadrik einer Geraden bei einer Projektion mit dem Netz $\mathcal{N}$. Eine an die Netzprojektion angeschlossene stereographische Projektion auf eine Quadrik $U$ und eine Polarität ergeben dann insgesamt eine Abbildung der Geraden des $\mathbb{P}^{3}$ auf die Punkte des $\mathbb{P}^{3}$.

Zur Übertragung der Konstruktion des linearen Bildes eines Punktes auf die Konstruktion des linearen Bildes einer Geraden behandeln wir die vier Typen linearer Geradenabbildungen $\mathcal{L} \rightarrow \mathbb{P}^{3}$ getrennt:

\subsubsection{Der elliptische Typ}

Als Zentrum $Z$ der linearen Abbildung $\lambda: \mathcal{L} \rightarrow \mathbb{P}^{3}$ wählen wir die Gerade

$$
Z=\left(t_{0}: t_{1}: 0: t_{0}: t_{1}: 0\right)
$$

des $\mathbb{P}^{5}$. Jede andere Gerade des $\mathbb{P}^{5}$, die $M_{2}^{4}$ in einem Paar konjugiert komplexer Punkte schneidet, führt zu einer projektiv äquivalenten linearen Abbildung. Ein zu $Z$ komplementärer Unterraum von $\mathbb{P}^{5}$ möge als Bildraum dienen. Es stellt keinerlei Einschränkung der Allgemeinheit dar, den Polarraum

$$
\widehat{Z}: x_{1}+x_{4}=x_{2}+x_{5}=0
$$

von $Z$ bezüglich $M_{2}^{4}$ als Bildraum der linearen Abbildung $\lambda \mathrm{zu}$ wählen. Damit wird der KLEINsche Bildpunkt einer Geraden $G=\left(g_{1}: \ldots: g_{6}\right)$ unter $\lambda$ auf

$$
G \lambda=\left(g_{1}-g_{4}: g_{2}-g_{5}: 2 g_{3}: 2 g_{6}\right)
$$

abgebildet.

Der Bildraum $\widehat{Z}$ schneidet $M_{2}^{4}$ nach der ovalen Quadrik

$$
U: x_{1}^{2}+x_{2}^{2}-x_{3} x_{6}=0 .
$$




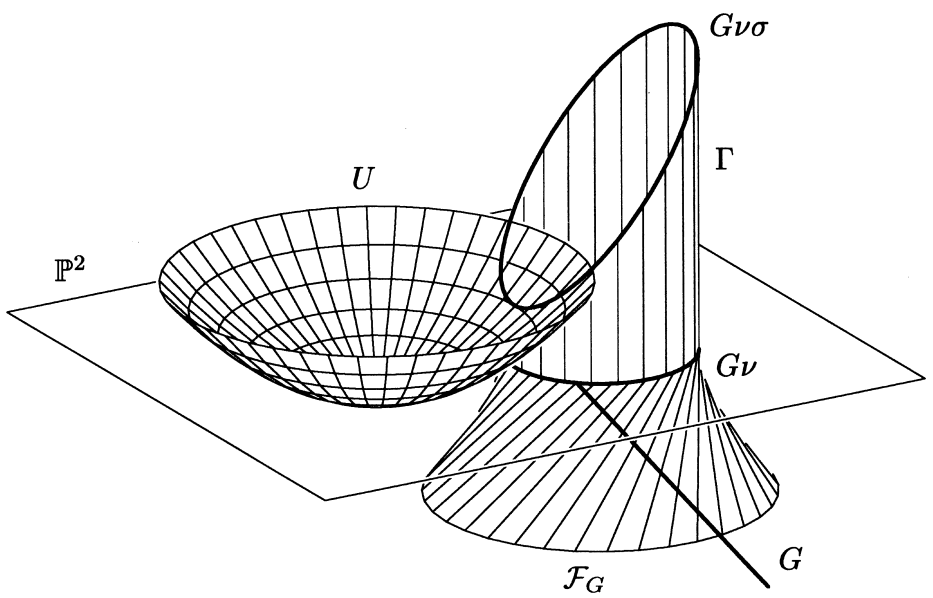

Abb. 2. Zerlegung der elliptischen linearen Geradenabbildung

Die Punkte von $U$ sind die KLEINschen Bilder der Geraden eines elliptischen Netzes $\mathcal{N}$ mit den Achsen $\left\{A_{1}, A_{2}\right\}=\left(Z \cap M_{2}^{4}\right) \gamma^{-1}$ mit $A_{1}=(1: i: 0: 1: i: 0)$ und $A_{2}=(1:-i: 0: 1:-i: 0)$.

Wir konstruieren nun das lineare Bild einer Geraden $G=\left(g_{1}: \ldots: g_{6}\right) \mathrm{im} \mathbb{P}^{3}$, indem wir sie zunächst mittels $\mathcal{N}$ gemäß (2) in die Ebene $\mathbb{P}^{2}: x_{3}=0$ projizieren. Ihr Netzriß ist der Kegelschnitt

$G \nu: g_{6} x_{0}^{2}-\left(g_{2}-g_{5}\right) x_{0} x_{1}+\left(g_{1}-g_{4}\right) x_{0} x_{2}+g_{3}\left(x_{1}^{2}+x_{2}^{2}\right)=x_{3}=0$.

Die stereographische Projektion $\sigma: \mathbb{P}^{2} \rightarrow U \backslash S$ mit Zentrum $S=(0: 0: 0: 1)$ bildet den Kegelschnitt (9) auf einen Kegelschnitt $G \nu \sigma$ in der Ebene

$$
g_{6} x_{0}-\left(g_{2}-g_{5}\right) x_{1}+\left(g_{1}-g_{4}\right) x_{2}+g_{3} x_{3}=0
$$

ab, da sich von der Schnittkurve des Projektionskegels $\Gamma:=S \vee G \nu$ und $U$ die zwei Erzeugenden $T_{S} U \cap U$ in der Tangentialebene $T_{S} U$ von $U$ in $S$ abspalten. Im Vergleich von (10) mit der Polarform

$$
u_{3} x_{6}+2 u_{1} x_{1}+2 u_{2} x_{2}+u_{6} x_{3}=0
$$

von (8) findet man den Pol

$$
G \nu \sigma \pi=\left(2 g_{6}: g_{5}-g_{2}: g_{1}-g_{4}: 2 g_{6}\right)
$$

der Trägerebene von $G \nu \sigma$. Vergleichen wir die Abbildungsgleichungen (7) von $G \mapsto G \lambda$ mit jenen in (12) von $G \mapsto G \nu \sigma \pi$, so stellen wir 
fest, daß die Abbildungen $\lambda$ und $\nu \sigma \pi$ bis auf eine Kollineation übereinstimmen. Es gilt also:

Satz 3.1. Die linearen Geradenabbildungen $\mathcal{L} \rightarrow \mathbb{P}^{3}$ vom elliptischen Typ können im $\mathbb{P}^{3}$ durch die Zusammensetzung einer Netzprojektion mit einem elliptischen Netz, einer stereographischen Projektion auf eine ovale Quadrik $U$ und der Polarität an $U$ realisiert werden.

Die Quadrik $U$ mit der Gleichung (8) kann man im Sinne der darstellenden Geometrie auch als Umriß der PLÜCKER-Quadrik $M_{2}^{4}$ (1) auffassen.

\subsubsection{Eine HOPF-Abbildung als lineare Geradenabbildung}

Wir betrachten die stereographische Projektion $\sigma: \mathbb{P}^{2} \rightarrow S^{2} \backslash N$ mit dem Zentrum $N=(1: 0: 0: 1)$ aus der Ebene $\mathbb{P}^{2}: x_{3}=0$ in die euklidische Einheitssphäre $S^{2}$. Unter Verwendung von homogenen Koordinaten $\left(x_{0}: x_{1}: x_{2}: x_{3}\right)$ für Punkte in $\mathbb{P}^{2}$ erhalten wir die Abbildungsgleichungen

$$
\sigma: \quad\left(x_{0}: x_{1}: x_{2}\right)=\left(x_{0}^{2}+x_{1}^{2}+x_{2}^{2}: 2 x_{0} x_{1}: 2 x_{0} x_{2}: x_{1}^{2}+x_{2}^{2}-x_{0}^{2}\right) .
$$

Schließt man nun $\mathbb{P}^{2}$ konform ab, indem man das uneigentliche Element $\infty$ ihrer Punktmenge hinzufügt, und erklärt man darüber hinaus $N \sigma^{-1}=\infty$, so ist eine bijektive Abbildung zwischen $\mathbb{P}^{2}$ und $S^{2}$ hergestellt, die Geraden und Kreise in $\mathbb{P}^{2}$ in Kreise auf $S^{2}$ überführt.

Ist ferner $\nu$ die Projektion (2) mittels elliptischem Netz in die Ebene $\mathbb{P}^{2}$, so ist die Abbildung $\phi=\nu \sigma$, die einen Punkt $P=\left(p_{0}: p_{1}: p_{2}: p_{3}\right) \in \mathbb{P}^{3}$ auf den Punkt

$$
P \phi=\left[\begin{array}{c}
p_{0}^{2}+p_{1}^{2}+p_{2}^{2}+p_{3}^{2} \\
2\left(p_{0} p_{1}+p_{2} p_{3}\right) \\
2\left(p_{0} p_{2}-p_{1} p_{3}\right) \\
-p_{0}^{2}+p_{1}^{2}+p_{2}^{2}+p_{3}^{2}
\end{array}\right]
$$

abbildet, eine HoPF-Abbildung. Nützt man die HoPF-Abbildung (14), um die Punkte einer Geraden $G=\left(g_{1}: \ldots: g_{6}\right)$ abzubilden, so findet man diese Bildpunkte auf $S^{2}$ in der Ebene

$$
x_{0}\left(g_{3}+g_{6}\right)+x_{1}\left(g_{2}-g_{5}\right)+x_{2}\left(g_{4}-g_{1}\right)+x_{3}\left(g_{6}-g_{3}\right)=0
$$


wieder. Der Vergleich mit der Polarform

$$
-x_{0} u_{0}+x_{1} u_{1}+x_{2} u_{2}+x_{3} u_{3}=0
$$

von $S^{2}$ liefert mit

$$
\left(g_{3}+g_{6}: g_{5}-g_{2}: g_{1}-g_{4}: g_{3}-g_{6}\right)
$$

den Pol dieser Ebene bezüglich $S^{2}$. Damit ist ganz offensichtlich ein Zusammenhang zwischen der HoPF-Abbildung und dem elliptischen Typ linearer Geradenabbildungen hergestellt. Es gilt also:

Satz 3.2. Die HOPF-Abbildung $\phi(14)$ ist eine lineare Geradenabbildung $\mathcal{L} \rightarrow \mathbb{P}^{3 \star}$.

Die Zusammensetzung der HoPF-Abbildung $\phi$ (14) und des Polarsystems an der euklidischen Einheitssphäre $S^{2}$ ist eine lineare Geradenabbildung $\mathcal{L} \rightarrow \mathbb{P}^{3}$.

\subsubsection{Der hyperbolische Typ}

Als Zentrum $Z$ der linearen Abbildung $\lambda: \mathbb{P}^{5} \rightarrow \mathbb{P}^{3}$ wählen wir nun die Gerade

$$
Z=\left(0: 0: t_{0}: 0: 0: t_{1}\right)
$$

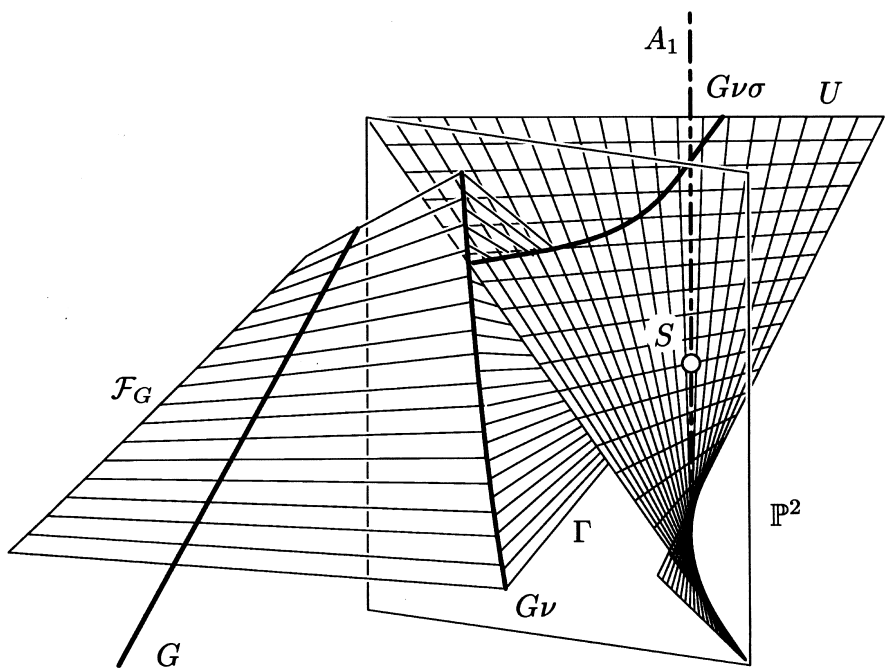

Abb. 3. Zerlegung der hyperbolischen linearen Geradenabbildung 
Die Wahl jeder anderen Sekante der PLÜCKER-Quadrik liefert eine projektiv äquivalente lineare Geradenabbildung. Der zu $Z$ komplementäre Bildraum soll auch in diesem Fall der Polarraum $\widehat{Z}$ von $Z$ bezüglich $M_{2}^{4}$ sein. Er ist durch die Gleichungen

$$
\widehat{Z}: x_{3}=x_{6}=0
$$

bestimmt. Das KLEINsche Bild einer Geraden $G$ mit den PLÜCKERKoordinaten $\left(g_{1}: \ldots: g_{6}\right)$ wird dann unter der so bestimmten linearen Abbildung $\lambda: \mathbb{P}^{5} \rightarrow \mathbb{P}^{3}$ auf den Punkt

$$
G \lambda=\left(g_{1}: g_{2}: g_{4}: g_{5}\right)
$$

abgebildet.

Der Bildraum $\widehat{Z}$ schneidet $M_{2}^{4}$ nach der ringartigen Quadrik

$$
U: \quad x_{1} x_{4}+x_{2} x_{5}=0 .
$$

Die Punkte von $U$ sind die KLEINschen Bilder der Geraden eines hyperbolischen Netzes $\mathcal{N}$ mit den Achsen $\left\{A_{1}, A_{2}\right\}=\left(Z \cap M_{2}^{4}\right) \gamma^{-1}$ mit $A_{1}=(0: 0: 1: 0: 0: 0)$ und $A_{2}=(0: 0: 0: 0: 0: 1)$.

Wir schreiten zur Konstruktion des linearen Bildes von $G$ in $\mathbb{P}^{3}$. Die Gerade $G=\left(g_{1}: \ldots: g_{6}\right)$ wird nun vermöge der Projektion (3) mit dem Netz $\mathcal{N}$ in die Ebene $\mathbb{P}^{2}: x_{0}=x_{1}$ auf den Kegelschnitt

$$
G \nu: \quad g_{4} x_{1}^{2}+g_{5} x_{1} x_{2}+g_{2} x_{1} x_{3}-g_{1} x_{2} x_{3}=x_{0}-x_{1}=0
$$

abgebildet. Die stereographische Projektion $\sigma: \mathbb{P}^{2} \rightarrow U \backslash S$ mit dem Zentrum $S=(1: 0: 0: 0)$ führt den Kegelschnitt $G \nu$ (22) in den Kegelschnitt $G \nu \sigma$ auf $U$ über, da sich auch in diesem Falle von der Schnittkurve des Projektionskegels $\Gamma:=S \vee G \nu$ und der Quadrik $U$ die zwei in der Tangentialebene $T_{S} U$ gelegenen Erzeugenden abspalten. Die Trägerebene des Kegelschnitts $G \nu \sigma$ hat die Gleichung

$$
l_{1} x_{0}+l_{4} x_{1}+l_{5} x_{2}+l_{2} x_{3}=0 .
$$

Der Vergleich mit der Polarform von $U$

$$
u_{1} x_{0}+u_{0} x_{1}+u_{3} x_{2}+u_{2} x_{3}=0
$$

lehrt uns, daß die Polarität $\pi$ an $U$ der Ebene (23) den Punkt

$$
G \nu \sigma \pi=\left(g_{4}: g_{1}: g_{2}: g_{5}\right)
$$

zuordnet. Auch hier stellen wir sofort fest, daß die Bilder (20) und (25) nicht nur linear in den PLÜCKER-Koordinaten sind, sondern auch nach geeignetem Permutieren der Koordinaten aus einander hervor- 
gehen, was einer Kollineation des Bildraumes entspricht. Wie im Falle der elliptischen linearen Geradenabbildungen haben wir also:

Satz 3.3. Die linearen Geradenabbildungen $\mathcal{L} \rightarrow \mathbb{P}^{3}$ vom hyperbolischen Typ können im $\mathbb{P}^{3}$ durch die Zusammensetzung einer Netzprojektion mit einem hyperbolischen Netz, einer stereographischen Projektion auf eine ringartige Quadrik $U$ und der Polarität an $U$ realisiert werden.

Wie im vorangegangenen Falle kann auch hier die Quadrik $U$ als Umriß der PLÜCKER-Quadrik $M_{2}^{4}$ bei der linearen Abbildung $\lambda$ beziehungsweise bei $\nu \sigma \pi$ aufgefaßt werden.

Die Zusammensetzung der Netzprojektion und der anschließenden stereographischen Projektion ergibt im hyperbolischen wie im elliptischen Fall eine verallgemeinerte stereographische Projektion, wie sie in [6] und [13] zur Anwendung kam.

\subsubsection{Der parabolische Typ}

Als Zentrum $Z$ der Projektion $\lambda: \mathbb{P}^{5} \rightarrow \mathbb{P}^{3}$ ist nun eine Tangente von $M_{2}^{4}$ zu wählen. Es sei daher

$$
Z=\left(0: t_{0}: 0: t_{1}: t_{0}: 0\right) .
$$

Der Polarraum $\widehat{Z}$ von $Z$ bezüglich $M_{2}^{4}$ ist dann durch

$$
\widehat{Z}: \quad x_{1}=x_{2}+x_{5}=0
$$

bestimmt und nicht als Bildraum der Projektion aus $Z$ geeignet, da $Z$ und $\widehat{Z}$ den Punkt $(0: 0: 0: 1: 0: 0)$ gemein haben, also nicht komplementär sind. Wir wählen daher

$$
\widetilde{Z}: \quad x_{4}=x_{5}=0
$$

als Bildraum. Die Abbildung $\lambda: \mathbb{P}^{5} \backslash Z \rightarrow \mathbb{P}^{3}$ kann somit durch die in den PLÜCKER-Koordinaten $\left(g_{1}: \ldots: g_{6}\right)$ einer Geraden $G$ linearen Abbildungsgleichungen

$$
G \lambda=\left(g_{1}: g_{2}-g_{5}: g_{3}: g_{6}\right)
$$

beschrieben werden.

Der Polarraum $\widehat{Z}$ von $Z$ schneidet $M_{2}^{4}$ nach dem quadratischen Kegel

$$
U: \quad x_{3} x_{6}-x_{2}^{2}=0,
$$




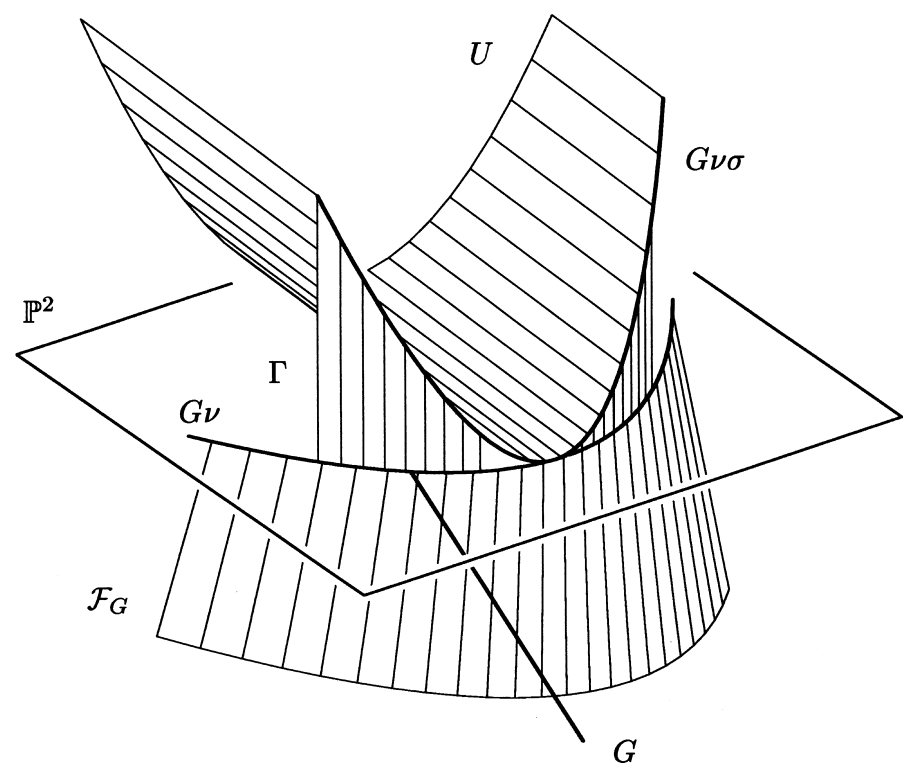

Abb. 4. Zerlegung der parabolischen linearen Geradenabbildung

dessen Punkte die KLEINschen Bilder der Geraden eines parabolischen Netzes $\mathcal{N}$ sind. Die Achse $A$ ist das $\gamma^{-1}$-Bild der Spitze von $U$ beziehungsweise das $\gamma^{-1}$-Bild des Berührpunktes von $Z$ mit $M_{2}^{4}$.

Wie in den beiden vorangegangenen Fällen ermitteln wir nun das lineare Bild einer Geraden $G$ im $\mathbb{P}^{3}$. Wir bestimmen den Netzriß $G \nu$ der Geraden $G=\left(g_{1}: \ldots: g_{6}\right)$ mit Hilfe von (4) in die Ebene $x_{3}=0$ und erhalten den Kegelschnitt

$$
G \nu: \quad g_{6} x_{0}^{2}+\left(g_{5}-g_{2}\right) x_{0} x_{1}+g_{1} x_{0} x_{2}+g_{3} x_{1}^{2}-g_{1} x_{1} x_{3}=0 .
$$

Sodann erfolgt die stereographische Projektion $\sigma: \mathbb{P}^{2} \rightarrow U \backslash S$ mit dem Zentrum $S=(0: 0: 0: 1)$. Dabei wird der Kegelschnitt $G \nu$ auf den Kegelschnitt $G \nu \sigma$ auf dem quadratischen Kegel $U$ abgebildet, da sich von der Schnittkurve des Projektionskegels $\Gamma:=S \vee G \nu$ mit $U$ auch hier die Erzeugenden in $T_{S} U$ abspalten.

Die Trägerebene des Kegelschnittes $G \nu \sigma$ ist durch

$$
l_{6} x_{0}+\left(l_{5}-l_{2}\right) x_{1}+l_{1} x_{2}+l_{3} x_{3}=0
$$

gegeben. Da die Quadrik $U$ mit der Gleichung (30) singulär ist, gilt dies auch für ihr Polarsystem. Es bestimmt keine Korrelation 
$\mathbb{P}^{3 \star} \rightarrow \mathbb{P}^{3}$. Um aber zu einer bijektiven Abbildung der Ebenen (32) auf die Punkte des $\mathbb{P}^{3}$ zu gelangen, können wir irgendeine Polarität oder Nullpolarität $\delta$ anwenden. Diese ist dann sicher linear in den homogenen Koordinaten von (32) und damit auch in den PLÜCKERKoordinaten von $G$. Damit haben wir insgesamt:

Satz 3.4. Die linearen Geradenabbildungen $\mathcal{L} \rightarrow \mathbb{P}^{3}$ vom parabolischen Typ können im $\mathbb{P}^{3}$ durch die Zusammensetzung einer Netzprojektion mit einem parabolischen Netz, einer stereographischen Projektion auf einen quadratischen Kegel und einer Polarität oder Nullpolarität realisiert werden.

Die Quadrik $U$ kann auch hier als der Umriß von $M_{2}^{4}$ angesehen werden. Auch bei der Konstruktion einer linearen Geradenabbildung vom parabolischen Typ kann man die Projektion mittels parabolischem Netz und die anschließende stereographische Projektion zur verallgemeinerten stereographischen Projektion zusammenfassen.

Die Erzeugung der elliptischen, hyperbolischen und parabolischen linearen Geradenabbildung kann somit einheitlich gesehen werden. Es gilt deshalb:

Satz 3.5. Die verallgemeinerte stereographische Projektion vermittelt eine lineare Geradenabbildung $\mathcal{L} \rightarrow \mathbb{P}^{3 \star}$. Die Zusammensetzung mit einer Korrelation liefert insgesamt eine lineare Geradenabbildung $\mathcal{L} \rightarrow \mathbb{P}^{3}$, die den Geraden des $\mathbb{P}^{3}$ Punkte des $\mathbb{P}^{3}$ zuordnet.

\subsubsection{Der singuläre Typ}

Der letzte noch zu diskutierende Typ linearer Geradenabbildungen $\mathcal{L} \rightarrow \mathbb{P}^{3}$ ist der singuläre. Hierbei kann als Zentrum die ganz in $M_{2}^{4}$ gelegene Gerade

$$
Z=\left(0: 0: 0: 0: t_{0}: t_{1}\right)
$$

gewählt werden. Jede andere in $M_{2}^{4}$ enthaltene Gerade kann durch eine automorphe Kollineation von $M_{2}^{4}$ auf diese abgebildet werden und liefert somit eine projektiv äquivalente lineare Abbildung $\lambda: \mathbb{P}^{5} \backslash Z \rightarrow \mathbb{P}^{3}$.

Der Polarraum von $Z$ ist nun durch die Gleichungen

$$
\widehat{Z}: x_{2}=x_{3}=0
$$

bestimmt und taugt aus demselben Grund wie im Falle der parabolischen linearen Geradenabbildung nicht als Bildraum. Die 
Umrißquadrik $U=M_{2}^{4} \cap \widehat{Z}$ besteht nun aus dem in $Z$ koaxialen Ebenenpaar

$$
x_{1}=x_{2}=x_{3}=0 \quad \text { und } \quad x_{2}=x_{3}=x_{4}=0 .
$$

Wir wählen daher

$$
\mathbb{P}^{3}: x_{5}=x_{6}=0
$$

als Bildraum der linearen Abbildung $\lambda: \mathbb{P}^{5} \backslash Z \rightarrow \mathbb{P}^{3}$. Die Abbildungsgleichungen lauten dann

$$
G \lambda=\left(g_{1}: g_{2}: g_{3}: g_{4}\right) .
$$

Die Übertragung dieses Typs linearer Abbildungen in den $\mathbb{P}^{3}$ benötigt folgende Feststellung: Das Ebenenpaar (35) ist das KLEINsche Bild eines Bündels und eines Feldes von Geraden des $\mathbb{P}^{3}$. Eine geometrische Realisierung des singulären Typs linearer Geradenabbildungen in den $\mathbb{P}^{3}$ steht noch aus.

Ein Blick auf die Gleichungen (7), (20), (29) und (37) zeigt, daß diese linearen Geradenabbildungen surjektiv sind. Es gilt viel allgemeiner der folgende Satz:

Satz 3.6. Die surjektiven linearen Geradenabbildungen $\mu: \mathcal{L} \rightarrow \mathbb{P}^{3}$ vom elliptischen, parabolischen und hyperbolischen Typ können stets als Zusammensetzung einer Netzprojektion, einer stereographischen Projektion und einer anschließenden Polarität im $\mathbb{P}^{3}$ realisiert werden.

Beweis. Zum Nachweis der Zerlegbarkeit der linearen Geradenabbildungen genügt es zu zeigen, daß zu allen Punkten des $\mathbb{P}^{3}$ in jedem Falle mindestens ein Urbild (eine Urbildgerade) gefunden werden kann. $\mathrm{Zu}$ diesem Zweck sind die oben angegebenen Konstruktionen lediglich umzukehren.

1. Der hyperbolische und der elliptische Fall: Liegt eine hyperbolische oder eine elliptische lineare Geradenabbildung vor, so existiert stets eine reguläre Quadrik $U$ als Umriß von $M_{2}^{4}$. Bei einer Abbildung hyperbolischen Typs handelt es sich um eine ringartige, andernfalls um eine ovale Quadrik.

Das Polarsystem an $U$ ist in beiden Fällen reell und bildet die Punkte $X \in \mathbb{P}^{3}$ bijektiv auf die Ebenen des $\xi \subset \mathbb{P}^{3}$ ab. Die Ebenen $\xi$ schneiden die Quadrik $U$ nach einem Kegelschnitt oder berühren diese, weshalb hier zwei Fälle zu unterscheiden sind:

(a) Die Ebene $\xi$ schneidet $U$ : Schneidet die Ebene $\xi$ die Quadrik $U$ nach einem Kegelschnitt $k_{X}$, so geht dieser durch stereographische 
Projektion $\sigma^{-1}$ aus einem geeigneten Zentrum $S \in U$ in eine Ebene $\mathbb{P}^{2} \neq T_{S} U$ aus bekannten Gründen in einen Kegelschnitt $k_{X} \sigma^{-1}$ über. Die Bildkegelschnitte aller in $U$ enthaltenen Kegelschnitte haben die beiden Punkte $\left\{P_{1}, P_{2}\right\}:=T_{S} U \cap U \cap \mathbb{P}^{2}$ (die Schnittpunkte der in der Tangentialebene des Projektionszentrums gelegenen Erzeugenden von $U$ mit $\left.\mathbb{P}^{2}\right)$ gemein. Das Punktepaar $\left\{P_{1}, P_{2}\right\}$ fällt bei Vorgabe einer elliptischen linearen Geradenabbildung konjugiert komplex, bei einer hyperbolischen reell aus.

Der Kegelschnitt $k_{X} \sigma^{-1}$ ist nun leicht als Netzriß einer Geraden zu deuten. Man wähle hierzu die Achsen $A_{i}$ eines elliptischen oder hyperbolischen Netzes $\mathcal{N}$, entsprechend dem Typ der linearen Geradenabbildung, je eine dieser Achsen durch die Punkte $P_{i}$. Durch den Kegelschnitt $k_{X} \sigma^{-1}$ und das Paar windschiefer Geraden $\left(A_{1}, A_{2}\right)$, das mit den Kegelschnitten $k_{x} \sigma^{-1}$ das Punktepaar $\left(P_{1}, P_{2}\right)$ teilt, ist die Faserquadrik $\mathcal{F}_{X}$ zu einem Urbild des Punktes $X$ eindeutig bestimmt. Als Urbild des Punktes $X$ kommt somit jede Gerade des ergänzenden Regulus (das ist jener, $\operatorname{dem} A_{i}$ nicht angehören) auf $\mathcal{F}_{X}$ in Frage.

(b) Die Ebene $\xi$ berührt $U$ : Berührt die Ebene $\xi$ die Quadrik $U$, so ist $X$ ein Punkt von $U$. Ferner sind dann auch $k_{X}$ und $k_{X} \sigma^{-1}$ Punkte. Letzterer ist Netzriß der aus ihm an die Achsen $A_{1}$ und $A_{2}$ legbaren Treffgerade, womit in diesem Fall das Urbild von $X$ sogar eindeutig als Gerade des Netzes $\mathcal{N}$ rekonstruiert werden kann.

2. Der parabolische Fall: Zur parabolischen linearen Geradenabbildung gehört ein quadratischer Kegel $U$ mit Spitze $V$ als Umriß von $M_{2}^{4}$. Das Polarsystem des quadratischen Kegels vermittelt keine bijektive Abbildung der Punkte des $\mathbb{P}^{3}$ auf die Ebenen des $\mathbb{P}^{3}$. Hier ist eine beliebige Polarität oder Nullpolarität $\delta$ anzuwenden, um dem Punkt $X \in \mathbb{P}^{3}$ die Ebene $X \delta=\xi$ zuzuordnen. Die Ebene $\xi$ schneidet $U$ nach einem Kegelschnitt $k_{X}$, welcher nach stereographischer Projektion $\sigma$ aus einem Punkt $S \in U$ in eine Ebene $\mathbb{P}^{2} \neq T_{S} U$ in einen Kegelschnitt $k_{X} \sigma^{-1}$ übergeht.

Die Kegelschnitte $k_{X} \sigma^{-1}$ bilden auch hier eine drei-parametrige Schar, denn sie haben alle ein Linienelement gemeinsam: Die gemeinsame Tangente $t$ ist die Spurgerade von $T_{S} U$ in $\mathbb{P}^{2}$, der gemeinsame Berührpunkt $P$ ist der Spurpunkt jener Erzeugenden von $U$, die durch $S$ geht.

Wählt man nun die Achse $A$ eines parabolischen Netzes durch $P$ und die Projektivität längs $A$ so, daß die gemeinsame Tangente $t$ aller Kegelschnitte $k_{X} \sigma^{-1}$ eine Netzgerade ist, dann sind die Faserquadriken $\mathcal{F}_{X}$ der Netzprojektion durch den Kegelschnitt $k_{X} \sigma^{-1}$, die Erzeugende $A$ und die Berührprojektivität längs $A$ 
eindeutig bestimmt. Die Urbildgeraden des Punktes $X$ sind dann alle Erzeugenden des ergänzenden Regulus (dieser enthält die Gerade $A$ nicht) auf $\mathcal{F}_{X}$.

Da im parabolischen Fall eine beliebige Polarität oder Nullpolarität zur Anwendung kommt, ist die Fallunterscheidung zwischen Punkten auf $U$ und solchen nicht auf $U$ bei der Rekonstruktion des Urbildes nicht mehr nötig. Im allgemeinen werden die Punkte von $U$ nicht auf ihre Tangentialebenen abgebildet werden.

Der singuläre Fall hat zwar kein Recht, an dieser Stelle behandelt zu werden, dennoch liegt seine Surjektivität auf der Hand. Man betrachte hierzu die Abbildungsgleichungen (37).

Weiters sei noch bemerkt, daß die bei der Rekonstruktion der Urbildgeraden verwendete Korrelation $\delta$ bestimmten Punkten $X$ eine Ebene $\xi$ durch die Kegelspitze $V$ zuordnet. (Die Menge all dieser Punkte erfüllt eine Ebene.) Die Ebene $\xi$ schneidet $U$ in einem nichtnotwendig reellen Paar von Erzeugenden, die vermöge $\sigma^{-1}$ auf Geraden in $\mathbb{P}^{2}$ abgebildet werden. Diese stimmen dann mit Urbildern unter der Netzprojektion überein.

In allen drei Typen nicht singulärer linearer Geradenabbildungen $\mathcal{L} \rightarrow \mathbb{P}^{3}$ treten die reellen Innenpunkte der Quadrik $U$ als die linearen Bilder nicht reeller Geraden auf.

\section{Beziehungen zu bekannten linearen Geradenabbildungen}

\subsection{Lineare Geradenabbildungen in eine projektive Ebene}

Wir stellen uns in diesem Abschnitt die Frage: In den zweidimensionalen Zentren $Z_{\mu}$ welcher linearer Geradenabbildungen $\mu: \mathcal{L} \rightarrow \mathbb{P}^{2}$ können wir die ein-dimensionalen Zentren $Z_{\lambda}$ welcher Typen linearer Geradenabbildungen $\lambda: \mathcal{L} \rightarrow \mathbb{P}^{3}$ wieder finden?

Wir folgen der in [4] vorgenommenen Einteilung der linearen Geradenabbildungen $\mu: \mathcal{L} \rightarrow \mathbb{P}^{2}$.

1. $Z_{\mu} \cap M_{2}^{4}=: k$ ist ein reeller Kegelschnitt: Der Kegelschnitt $k$ ist KLEINsches Bild eines Regulus einer ringartigen Quadrik. Folglich können in $Z_{\mu}$ Geraden $Z_{\lambda}$ als Zentren linearer Geradenabbildungen $\mathcal{L} \rightarrow \mathbb{P}^{3}$ gewählt werden, die $k$ entweder in einem reellen Punktepaar schneiden, berühren oder in einem Paar konjugiert komplexer Punkte schneiden. Es sind daher die Zentren linearer Geradenabbildungen vom elliptischen, parabolischen und hyperbolischen Typ in $Z_{\mu}$ wählbar. Eine Gerade $Z_{\lambda} \subset M_{2}^{4}$, die als Zentrum einer singulären 
linearen Geradenabbildung dienen kann, existiert in $Z_{\mu}$ naturgemäß nicht.

2. $Z_{\mu} \cap M_{2}^{4}=: k$ ist ein nullteiliger Kegelschnitt: Der Kegelschnitt $k$ ist KLEINsches Bild eines Regulus einer ovalen Quadrik des $\mathbb{P}^{3}$ und trägt keinen reellen Punkt. Deshalb kann in der Ebene $Z_{\mu}$ keine Gerade $Z_{\lambda}$ gefunden werden, die $k$ und damit $M_{2}^{4}$ nach einem reellen Punktepaar schneidet oder $k$ und damit auch $M_{2}^{4}$ in einem reellen Punkt berührt. Es können in $Z_{\mu}$ nur Geraden $Z_{\lambda}$ gewählt werden, die als Zentren linearer Geradenabbildungen des elliptischen Typs dienen. Auch in diesem Fall existiert keine Gerade $Z_{\lambda} \subset Z_{\mu}$, die auch $M_{2}^{4}$ angehört.

3. $Z_{\mu} \cap M_{2}^{4}=:\left\{e_{1}, e_{2}\right\}$ ist ein reelles Geradenpaar: In $Z_{\mu}$ existieren Geraden $Z_{\lambda}$, die $M_{2}^{4}$ nur im Punkt $e_{1} \cap e_{2}=: S$ schneiden, diese können als Zentren linearer Geradenabbildungen des parabolischen Typs verwendet werden. Darüber hinaus existieren auch Geraden $Z_{\lambda} \subset Z_{\mu}$, die $M_{2}^{4}$ in einem reellen Punktepaar $\left\{P_{1}, P_{2}\right\}$ schneiden. Man wähle hierzu $P_{i} \in e_{i} \backslash S$ und $Z_{\lambda}=S_{1} \vee S_{2}$, welche dann als Zentren linearer Geradenabbildungen vom hyperbolischen Typ dienen können. Darüber hinaus kann auch jede der Geraden $e_{i}$ als Zentrum einer singulären linearen Geradenabbildung dienen.

4. $Z_{\mu} \cap M_{2}^{4}=\{e, \bar{e}\}$ ist ein Paar konjugiert komplexer Geraden: Der Schnittpunkt $S=e \cap \bar{e}$ ist sicher reell, weshalb man in diesem Fall Geraden $Z_{\lambda} \subset Z_{\mu}$ wählen kann, die mit $M_{2}^{4}$ nur den reellen Punkt $S$ gemein haben oder $M_{2}^{4}$ nach einem Paar konjugiert komplexer Punkte schneiden. Erstere führen zu linearen Geradenabbildungen des parabolischen, letztere zu solchen des elliptischen Typs.

5. $Z_{\mu} \cap M_{2}^{4}=e$ ist eine reelle Gerade: In diesen Ebenen existieren neben der Geraden $e$ nur solche, die $M_{2}^{4}$ in genau einem Punkt schneiden. Wählt man $e$ als Zentrum einer linearen Abbildung, dann handelt es sich um eine singuläre lineare Geradenabbildung, jede andere gehört zu einer Abbildung vom parabolischen Typ.

6. $Z_{\mu} \subset M_{2}^{4}$ ist eine Ebene der ersten Art: $\left(Z_{\mu}\right.$ ist KLEINsches Bild eines Geradenbündels in $\mathbb{P}^{3}$.) $\mathrm{Da} Z_{\mu}$ ganz in $M_{2}^{4}$ liegt, liegen alle ihre Geraden ganz in $M_{2}^{4}$. Hier können nur Zentren des singulären Typs linearer Geradenabbildungen gewählt werden.

7. $Z_{\mu} \subset M_{2}^{4}$ ist eine Ebene der zweiten Art: $\left(Z_{\mu}\right.$ ist KLEINsches Bild eines Geradenfeldes in $\mathbb{P}^{3}$.) Dieser Fall unterscheidet sich vom vorangegangenen nicht.

Wir bezeichnen der Einfachheit halber die sieben BRAUNERschen Typen linearer Geradenabbildungen $\mu: \mathcal{L} \rightarrow \mathbb{P}^{2}$ mit den Symbolen 
(1), .., (7) und die Typen linearer Geradenabbildungen $\lambda: \mathcal{L} \rightarrow \mathbb{P}^{3}$ mit den Symbolen $(e),(h),(p)$ und $(s)$ und können zusammenfassend folgendes Ergebnis aussprechen:

Satz 4.1. In den zweidimensionalen Zentren $Z_{\mu}$ linearer Geradenabbildungen $\mu: \mathcal{L} \rightarrow \mathbb{P}^{2}$ der Typen (1) bis (7) können die folgenden eindimensionalen Zentren $Z_{\lambda}$ linearer Geradenabbildungen der Typen (e), $(h),(p)$ und $(s)$ gefunden werden:

1. in (1) für die Typen $(e),(h)$ und $(p)$;

2. in (2) nur für den Typ (e),

3. in (3) für die Typen $(h),(p)$ und $(s)$;

4. in (4) für die Typen (e) und ( $p$ );

5. in (5) für die Typen $(p),(s)$;

6. in (6) nur für den Typ (s) und

7. in (7) nur für den Typ (s).

\subsection{Die kinematische Abbildung von BLASCHKE und GRÜNWALD}

Die kinematische Abbildung von W. BLASCHKE [2] und J. GRÜNWALD [10] ordnet den Geraden $G$ eines drei-dimensionalen quasielliptischen Raumes ein Punktepaar $\left(G^{l}, G^{r}\right)$ einer euklidischen Ebene $\mathbb{P}^{2}: x_{3}=0$ zu. Dazu wird $G$ mit den Ebenen $\varepsilon^{+}: x_{3}=x_{0}$ und $\varepsilon^{-}: x_{3}=-x_{0}$ geschnitten. Die Orthogonalprojektionen der Schnittpunkte $G^{+}$und $G^{-}$in die Ebene $\mathbb{P}^{2}$ werden dann um den Spurpunkt von $G$ in $\mathbb{P}^{2}$ um $+90^{\circ}$ gedreht und gelangen so in die Position $G^{r}$ und $G^{l}$, welche man linken und rechten Bildpunkt von $G$ nennt, siehe Abb. 5.

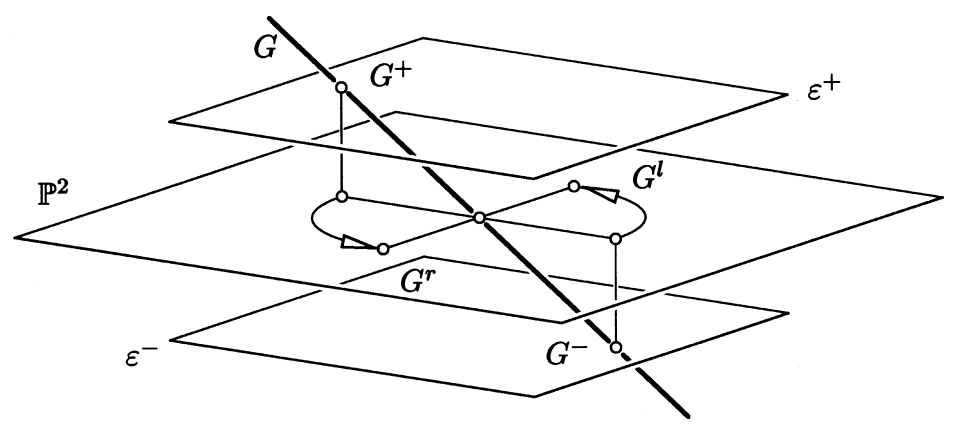

Abb. 5. Kinematische Abbildung von BLASCHKE und GrüNWALD 
Die Abbildungen $\phi^{l}: G \mapsto G^{l}$ und $\phi^{r}: G \mapsto G^{r}$ werden linke und rechte Abbildung genannt, durch

$G \phi^{l}=\left(g_{3}:-g_{2}-g_{4}: g_{1}-g_{5}\right)$ und $G \phi^{r}=\left(g_{3}: g_{2}-g_{4}:-g_{1}-g_{5}\right)$

beschrieben und sind offensichtlich lineare Geradenabbildungen $\mathcal{L} \rightarrow \mathbb{P}^{2}$. Fassen wir sie als lineare Abbildungen aus der PLÜCKERQuadrik $M_{2}^{4}$ auf, so sind die Ausnahmeräume $Z^{r}$ und $Z^{l}$ zwei Ebenen mit dem gemeinsamen Punkt $(0: 0: 0: 0: 0: 1)$, der KLEINsches Bild der Ferngeraden von $\mathbb{P}^{2}$ ist.

Betrachtet man die linke und die rechte Abbildung getrennt voneinander, so sind sie vom 6. beziehungsweise 7. BRAUNERschen Typ, siehe [4]. Man kann sie daher nur durch die Zusammensetzung des singulären Typs linearer Geradenabbildungen $\mathcal{L} \rightarrow \mathbb{P}^{3}$ und einer anschließenden Projektion erhalten.

\subsection{Die sphärisch kinematische Abbildung}

Die von W. K. CLIFFORD in [5] eingeführte sphärisch kinematische Abbildung ist eine lineare Geradenabbildung, die den orientierten Geraden $G$ des drei-dimensionalen elliptischen Raumes $\mathbb{E}^{3}$ ein Paar orientierter Geraden eines Bündels zuordnet.

$\mathrm{Da}$ die Geradenbündel des projektiven Raumes isomorph $\mathrm{zu}$ projektiven Ebenen sind, ist insgesamt eine Abbildung $\overrightarrow{\mathcal{L}} \rightarrow \mathbb{P}^{2} \times \mathbb{P}^{2}$ aus der Mannigfaltigkeit der orientierten Geraden des drei-dimensionalen elliptischen Raumes in ein Paar projektiver Ebenen hergestellt.

Man errechnet aus den PLÜCKER-Koordinaten $\left(g_{1}: \ldots: g_{6}\right)$ einer Geraden $G$ gemäß

$G^{l}=\left(g_{1}-g_{4}: g_{2}-g_{5}: g_{3}-g_{6}\right)$ und $G^{r}=\left(g_{1}+g_{4}: g_{2}+g_{5}: g_{3}+g_{6}\right)$

den linken und rechten Bildpunkt von $G$.

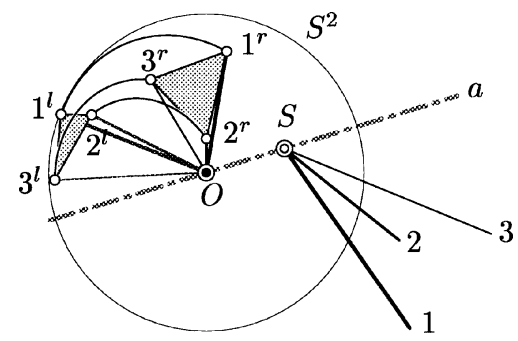

Abb. 6. Sphärisch kinematisches Bild eines Dreikants 
Die getrennte Betrachtung der linken und rechten Abbildung $\phi^{l}$ und $\phi^{r}$ liefert auch hier wieder Aufschluß über ihre Beziehungen zu den linearen Geradenabbildungen $\mathcal{L} \rightarrow \mathbb{P}^{3}$. Die Ausnahmeräume $Z^{l}$ und $Z^{r}$ sind Ebenen, die $M_{2}^{4}$ nach nullteiligen Kegelschnitten schneiden. Diese sind die KLEINschen Bilder der nicht reellen Ausnahmereguli der nullteiligen Quadrik

$$
x_{0}^{2}+x_{1}^{2}+x_{2}^{2}+x_{3}^{3}=0,
$$

welche auch als Maßquadrik des $\mathbb{E}^{3}$ dienen kann, siehe [9]. Da die Ausnahmeebenen keine reellen Punkte mit $M_{2}^{4}$ gemein haben, existieren in diesen auch nur Geraden, die $M_{2}^{4}$ nicht reell schneiden. Diese können mithin nur als Zentren der linearen Geradenabbildungen des elliptischen Typs dienen, weshalb die sphärisch kinematische Abbildung als Zusammensetzung einer linearen Geradenabbildung $\mathcal{L} \rightarrow \mathbb{P}^{3}$ des elliptischen Typs und einer anschließenden Projektion $\mathbb{P}^{3} \rightarrow \mathbb{P}^{2}$ erzeugt werden kann.

So kann etwa das linke Bild $G^{l}$ durch die Zusammensetzung aus der mit der Hopf-Abbildung (14) verbundenen linearen Geradenabbildung (17) und der Projektion aus $(1: 0: 0: 0)$ in die Ebene $x_{0}=0$ konstruiert werden.

Üblicherweise wird den PLÜCKER-Koordinaten $\left(g_{1}: \ldots: g_{6}\right)$ der Geraden des drei-dimensionalen elliptischen Raumes eine spezielle Normierung auferlegt, sodaß neben (1) auch

$$
g_{1}^{2}+g_{2}^{2}+g_{3}^{2}+g_{4}^{2}+g_{5}^{2}+g_{6}^{2}=1
$$

gilt. Mit dieser Normierung sind der linke und rechte Bildpunkt (39) Punkte der euklidischen Einheitssphäre $S^{2}$, weshalb man dann eine Abbildung $\overrightarrow{\mathcal{L}} \rightarrow S^{2} \times S^{2}$ erhält. Einem Dreikant orientierter Geraden des $\mathbb{E}^{3}$ entspricht ein Paar kongruenter sphärischer Dreiecke, also eine Drehung, die das linke Bild in das rechte überführt, siehe Abb. 6 .

Man spricht daher auch von der sphärisch kinematischen Abbildung, die ein recht nützliches Werkzeug in der Differentialund der Liniengeometrie des elliptischen Raumes $\mathbb{E}^{3}$ ist, siehe hierzu etwa [3] und [15].

\subsection{Die Abbildung von ECKHART und REHBOCK}

Die Geradenabbildungen, wie sie in $[7,8]$ und $[18,19]$ fast zur gleichen Zeit und unabhängig voneinander untersucht worden sind, sind zueinander duale Sichtweisen ein und desselben Abbildungsverfahrens für Geraden des $\mathbb{P}^{3}$. 

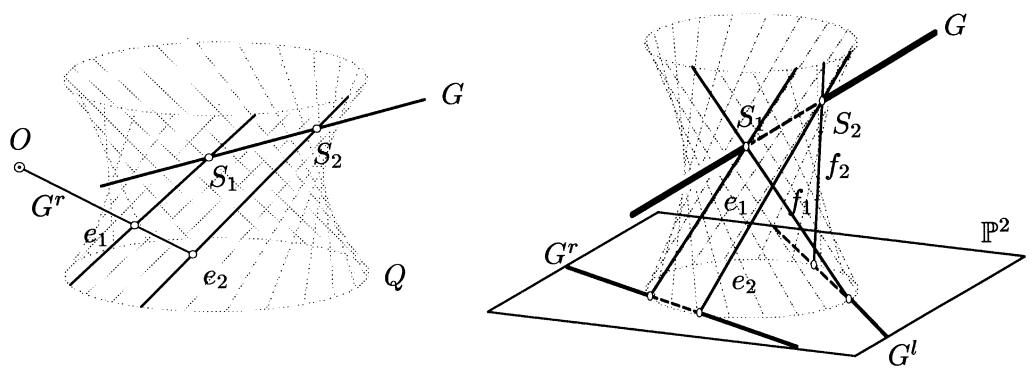

Abb. 7. Geradenabbildung nach ECKHART (links) und nach REHBOCK (rechts)

Die Geradenabbildung nach ECKHART $[7,8]$ bedarf einer Quadrik $Q$ und eines ausgezeichneten Punktes $O \notin Q$. Man ermittelt nun das linke und rechte Bild $G^{l}$ und $G^{r}$, indem man die Treffgeraden an die beiden Erzeugenden $e_{1}$ und $e_{2}$ der linken beziehungsweise $f_{1}$ und $f_{2}$ der rechten Schar durch die Schnittpunkte $S_{1}$ und $S_{2}$ von $G$ mit $Q$ aus $O$ legt, siehe Abb. 7.

Dual dazu kann man die Schnittpunkte von $e_{1}$ und $e_{2}$ mit einer Ebene $\mathbb{P}^{2}$ zum linken Bild $G^{l}$ einer Geraden $G$ verbinden. Die analoge Konstruktion mit den rechten Erzeugenden $f_{1}$ und $f_{2}$ ergeben das rechte Bild, siehe Abb. 7.

In [22] wurde unter Verwendung der nullteiligen Quadrik $Q$ mit der Gleichung (40) gezeigt, daß die linearen Risse auch mit ausschließlich linearen Konstruktionen ermittelt werden können. Die Beziehungen der linearen Geradenabbildungen von ECKHART und REHBOCK und der sphärisch kinematischen Abbildung zum Übertragungsprinzip von STUDY, wie es in [20] eingeführt wurde, ist offensichtlich.

\section{Geometrische Realisierung der linearen Geradenabbildungen in die projektive Ebene}

Die linearen Geradenabbildungen in den projektiven Raum $\mathbb{P}^{3}$ konnten, mit Ausnahme des singulären Typs, durch die Zusammensetzung einer Netzprojektion, einer stereographischen Projektion auf eine geeignete Quadrik und die Polarität an dieser realisiert werden.

An dieser Stelle sei bemerkt, daß die Netzprojektion alleine bereits eine lineare Geradenabbildung ist. Ihr Bildraum ist der projektive Raum der Kegelschnitte einer Ebene, die durch zwei feste Punkte gehen.

Auch die linearen Geradenabbildungen in die projektive Ebene $\mathbb{P}^{2}$ können direkt in $\mathbb{P}^{3}$ durch Hintereinanderausführen einer Netzpro- 
jektion $\nu: \mathcal{L} \rightarrow \mathbb{P}^{2}$ und einer anschließenden linearen Abbildung der Bildkegelschnitte auf die Punkte oder Geraden von $\mathbb{P}^{2}$ realisiert werden.

Dabei können aus den vier Typen linearer Geradenabbildungen $\lambda: \mathcal{L} \rightarrow \mathbb{P}^{3}$ die folgenden vier Typen gewonnen werden:

\subsection{Der elliptische Typ}

Die Realisierung des elliptischen Typs linearer Geradenabbildungen in den $\mathbb{P}^{2}$ sieht als ersten Schritt die Projektion mittels elliptischem Netz gemäß (2) vor. Jede nicht mit den Netzachsen zusammenfallende Gerade $G$ mit den PLÜCKER-Koordinaten $\left(g_{1}: \ldots: g_{6}\right)$ wird gemäß (2) auf den Kegelschnitt (9) abgebildet. Die Pole dieser Kegelschnitte bezüglich der Geraden $U: x_{0}=0 \subset \mathbb{P}^{2}$ sind dann

$$
G \nu \pi=\left(2 g_{3}: g_{2}-g_{5}: g_{4}-g_{1}\right) .
$$

Hierin steht $\pi: \mathbb{P}^{2 \star} \rightarrow \mathbb{P}^{2}$ für die Polbildung bezüglich $u$. Vergleicht man nun (7) und (41), so stellt man fest, das $G \lambda$ und $G \nu \pi$ bis auf eine Kollineation übereinstimmen. Damit ist die Komposition der Abbildungen $\nu$ und $\pi$ bis auf Kollineation eine lineare Geradenabbildung $\mathcal{L} \rightarrow \mathbb{P}^{2}$, und es gilt:

Satz 5.1. Die lineare Geradenabbildung $\mathcal{L} \rightarrow \mathbb{P}^{2}$ (41) ist Zusammensetzung einer linearen Geradenabbildung vom elliptischen Typ (7) und einer anschließenden linearen Abbildung $\mathbb{P}^{2} \rightarrow \mathbb{P}^{2}$. Sie wird als Zusammensetzung einer Netzprojektion und anschließender Polbildung des Netzrisses bezüglich einer festen Geraden $u \subset \mathbb{P}^{2}$ geometrisch realisiert.

\subsection{Der hyperbolische Typ}

Im Falle einer linearen Geradenabbildung vom hyperbolischen Typ wird eine Gerade $G$ mit Hilfe eines hyperbolischen Netzes gemäß (3) auf den Kegelschnitt (22) in $\mathbb{P}^{2}$ abgebildet, soferne $G$ nicht mit einer der Netzachsen zusammenfällt. Wir bestimmen auch hier die Pole bezüglich einer festen Geraden. Es sei $u$ : $x_{0}=x_{1}-x_{0}=0$, und wir erhalten

$$
G \nu \pi=\left(g_{1}: g_{2}: g_{5}\right),
$$

wobei auch hier das Symbol $\pi$ die Polbildung bezüglich $u$ bezeichne. Der Vergleich von (20) und (42) lehrt, daß auch hier die Zusammensetzung der Netzprojektion $\nu$ und der Polbildung $\pi$ mit der 
linearen Geradenabbildung (20) und der anschließenden Projektion in $\mathbb{P}^{2}$ bis auf eine Kollineation übereinstimmen. Auch hier gilt:

Satz 5.2. Die lineare Geradenabbildung $\mathcal{L} \rightarrow \mathbb{P}^{2}$ (42) ist Zusammensetzung einer linearen Geradenabbildung vom hyperbolischen Typ (20) und einer anschließenden linearen Abbildung $\mathbb{P}^{2} \rightarrow \mathbb{P}^{2}$. Sie wird als Zusammensetzung einer Netzprojektion und anschließender Polbildung des Netzrisses bezüglich einer festen Geraden $u \subset \mathbb{P}^{2}$ geometrisch realisiert.

\subsection{Der parabolische Typ}

Nun werden die Geraden $G$ des $\mathbb{P}^{3}$ unter Zuhilfenahme eines parabolischen Netzes gemäß (4) auf die Kegelschnitte (31) in $\mathbb{P}^{2}$ abgebildet. Ordnet man jedem dieser Kegelschnitte seine Polare bezüglich eines festen Punktes $U=(1: 0: 0) \in \mathbb{P}^{2}$ zu, so erhält man mit

$$
G \nu \pi^{\star}=\left(2 g_{6}: g_{5}-g_{2}: g_{1}\right)
$$

eine lineare Geradenabbildung $\mathcal{L} \rightarrow \mathbb{P}^{2 \star}$. Interpretiert man die homogenen Geradenkoordinaten (43) als Punktkoordinaten in einer projektiven Ebene, was der Anwendung einer Korrelation in $\mathbb{P}^{2 \star}$ gleichkommt, so liegt insgesamt eine lineare Geradenabbildung $\mathcal{L} \rightarrow \mathbb{P}^{2}$ vor. Auch die geometrische Realisierung dieser linearen Geradenabbildung ist somit gelungen. Es gilt daher:

Satz 5.3. Die lineare Geradenabbildung $\mathcal{L} \rightarrow \mathbb{P}^{2}$ (43) ist Zusammensetzung einer linearen Geradenabbildung vom parabolischen Typ (29) und einer anschließenden Korrelation $\mathbb{P}^{2 \star} \rightarrow \mathbb{P}^{2}$. Sie wird als Zusammensetzung einer Netzprojektion mit der Polarenbildung des Netzrisses bezüglich eines festen Punktes $U \subset \mathbb{P}^{2}$ und einer anschließenden Korrelation geometrisch realisiert.

\section{Zentrenwahl}

Wir stellen uns nun die Frage, ob durch die ein-dimensionalen Zentren der linearen Geradenabbildungen $\mathcal{L} \rightarrow \mathbb{P}^{3}$ zwei-dimensionale projektive Unterräume von $\mathbb{P}^{5}$ gewählt werden können, sodaß diese die Zentren der linearen Geradenabbildungen sind, wie sie in [4] klassifiziert wurden.

\subsection{Der elliptische Typ}

Von den sieben Typen linearer Geradenabbildungen $\lambda: \mathcal{L} \rightarrow \mathbb{P}^{2}$ können aus dem elliptischen Typ linearer Geradenabbildungen 
$\mu: \mathcal{L} \rightarrow \mathbb{P}^{3}$ drei gewonnen werden. Durch den ein-dimensionalen Ausnahmeraum $Z_{\lambda}$ können projektive Ebenen $Z_{\mu}$ so gewählt werden, daß sie $M_{2}^{4}$ nach einem reellen oder nullteiligen Kegelschnitt schneiden. Ferner existieren durch $Z_{\lambda}$ auch Ebenen $Z_{\mu}$, die $M_{2}^{4}$ nach einem Paar konjugiert komplexer Geraden schneiden. Damit sind die BRAUNERschen Typen (1), (2) und (4) durch Zusammensetzung einer linearen Geradenabbildung $\lambda: \mathcal{L} \rightarrow \mathbb{P}^{3}$ und einer linearen Abbildung $\pi: \mathbb{P}^{3} \rightarrow \mathbb{P}^{2}$ (Projektion + Kollineation) erzeugbar.

\subsection{Der hyperbolische Typ}

Schneidet $Z_{\lambda}$ die PLÜCKER-Quadrik in einem Paar reeller Punkte, so können Ebenen $Z_{\mu}$ durch $Z_{\lambda}$ gefunden werden, die $M_{2}^{4}$ nach einem reellen Kegelschnitt oder einem reellen Geradenpaar schneiden. Folglich ist es möglich, die BRAUNERschen Typen (1) und (3) linearer Geradenabbildungen $\mu: \mathcal{L} \rightarrow \mathbb{P}^{2}$ als Zusammensetzung einer linearen Geradenabbildung $\lambda: \mathcal{L} \rightarrow \mathbb{P}^{3}$ vom hyperbolischen Typ und einer linearen Abbildung $\pi: \mathbb{P}^{3} \rightarrow \mathbb{P}^{2}$ (Projektion + Kollineation) zu erzeugen.

\subsection{Der parabolische Typ}

Liegt mit $Z_{\lambda}$ eine Tangente von $M_{2}^{4}$ vor, so können durch diese Ebenen $Z_{\mu}$ gewählt werden, die $M_{2}^{4}$ nach einem reellen Kegelschnitt, einem reellen Geradenpaar oder genau einer reellen Geraden schneiden. Diese Ebenen dienen als Zentren linearer Geradenabbildungen $\mathcal{L} \rightarrow \mathbb{P}^{2}$ der BRAUNERschen Typen (1), (3) oder (5). Daher kann man diese Typen als Zusammensetzung einer linearen Geradenabbildung $\lambda: \mathcal{L} \rightarrow \mathbb{P}^{3}$ vom parabolischen Typ mit einer linearen Abbildung $\pi: \mathbb{P}^{3} \rightarrow \mathbb{P}^{2}$ (Projektion + Kollineation) erzeugen.

\subsection{Der singuläre Typ}

Im letzten Fall, der singulären linearen Geradenabbildung $\lambda: \mathcal{L} \rightarrow \mathbb{P}^{3}$ gilt $Z_{\lambda} \subset M_{2}^{4}$. In diesem Fall ist es möglich, zweidimensionale projektive Unterräume $Z_{\mu}$ durch $Z_{\lambda}$ so zu wählen, daß $Z_{\mu} \cap M_{2}^{4}$ entweder ein reelles Geradenpaar, genau eine reelle Gerade oder eine Ebene der ersten oder zweiten Art von $M_{2}^{4}$ ist. Demgemäß können die BRAUNERschen Typen (3), (5), (6) und (7) auch als Komposition einer singulären linearen Geradenabbildung $\lambda: \mathcal{L} \rightarrow \mathbb{P}^{3}$ und einer linearen Abbildung $\pi: \mathbb{P}^{3} \rightarrow \mathbb{P}^{2}$ (Projektion + Kollineation) gewonnen werden. 
Damit läßt sich zusammenfassend folgender Satz formulieren:

Satz 6.1. Aus den vier Typen linearer Geradenabbildungen $\lambda: \mathcal{L} \rightarrow \mathbb{P}^{3}$ ergeben sich nach anschließender Projektion $\pi: \mathbb{P}^{3} \rightarrow \mathbb{P}^{2}$ folgende linearen Geradenabbildungen der BRAUNERschen Typen:

1. Aus dem elliptischen Typ (e) erhält man die Typen (1), (2) und (4).

2. Aus dem hyperbolischen Typ (h) erhält man die Typen (1) und (3).

3. Aus dem parabolischen Typ ( $p$ ) erhält man die Typen (1), (3) und (5).

4. Aus dem singulären Typ (s) erhält man die Typen (3), (5), (6) und (7).

\section{Abschließende Betrachtungen}

In [4] sind die linearen Geradenabbildungen in projektive Ebenen klassifiziert worden. Es gibt je nach Lage des zwei-dimensionalen Zentrums $Z_{\mu}$ zur PLÜCKER-Quadrik sieben verschiedene Typen linearer Geradenabbildungen in eine projektive Ebene $\mathbb{P}^{2}$. Bei den in der vorliegenden Arbeit betrachteten surjektiven linearen Geradenabbildungen in den $\mathbb{P}^{3}$ waren vier Typen zu unterscheiden.

Neben den linearen Abbildungen mit ein- und zwei-dimensionalem Zentrum aus dem $\mathbb{P}^{5}$ in einen drei- beziehungsweise zweidimensionalen projektiven Raum sind aber noch jene mit einem null-dimensionalen (punktförmigen) Zentrum erwähnenswert. Hierbei wären dann zwei Typen linearer Geradenabbildungen zu unterscheiden, je nachdem, ob $Z \in M_{2}^{4}$ oder $Z \notin M_{2}^{4}$ gilt.

Liegt $Z \in M_{2}^{4}$, so handelt es sich bei der Projektion $\mathcal{L} \rightarrow \mathbb{P}^{4}$ um eine stereographische Projektion. Verwendet man eine geeignete Koordinatisierung der Geraden, so ist diese stereographische Projektion aus $M_{2}^{4}$ eine lineare Abbildung in einen $\mathbb{P}^{4}$. Eine rein geometrische Realisierung dieser Abbildung steht noch aus. Eine einfache Konstruktion des linearen Bildes einer Geraden unter Zuhilfenahme komplexer Zahlen hingegen ist möglich. Die stereographische Projektion aus $\mathcal{L}$ in $\mathbb{P}^{4}$ wurde bereits in [16] zum Zweck der Interpolation von Funktionen auf Geraden verwendet.

Der Fall eines drei-dimensionalen Zentrums führt zu einer sogenannten Kotierung, und es ist fragwürdig, was eine solche zum Studium oder zur Visualisierung von Geradenmannigfaltigkeiten beitragen könnte.

Die linearen Geradenabbildungen $\mathcal{L} \rightarrow \mathbb{P}^{3}$ können zur Visualisierung von Geradenmannigfaltigkeiten genützt werden. Dabei sei 
insbesondere an Geradenkongruenzen und die darin enthaltenen Regelflächen gedacht. Da die KLEINsche Abbildung nicht nur bilinear, sondern auch eine $C^{\infty}$-Abbildung ist und die linearen Geradenabbildungen diese Eigenschaften nicht zerstören, können differentialgeometrische Sachverhalte wiedergegeben werden.

\section{Danksagung}

Ein Wort des Dankes soll an dieser Stelle an all jene gerichtet sein, die am Zustandekommen dieser Arbeit beteiligt waren. Zum einen sei H. POTTMANN für den Hinweis gedankt, die linearen Geradenabbildungen zu studieren. Zum anderen danke ich H. STACHEL für seine konstruktive Kritik und so manche Anregung.

\section{Literatur}

[1] Bereis, R., BraunER, H. (1957) Schraubung und Netzprojektion. Elem. Math. 12: $34-40$

[2] BLASCHKE, W. (1911) Euklidische Kinematik und nichteuklidische Geometrie. Zeitschr. angew. Math. u. Phys. 60: 61-91, 203-204

[3] BlaschKe, W. (1960) Kinematik und Quaternionen. VEB Deutscher Verlag der Wissenschaften, Berlin

[4] BRAUnER, H. (1973) Eine geometrische Kennzeichnung linearer Geradenabbildungen. Mh. f. Math. 77: 10-20

[5] Clifford, W. K. (1873) Preliminary sketch on biquaternions. Proc. London Math. Soc. 4: 381-395

[6] DietZ, R., HoscheK, J., JÜTTLER, B. (1993) An algebraic approach to curves and surfaces on the sphere and other quadrics. Comp. Aid. Des. 10: 211-229

[7] ECKHART, L. (1923) Über die Abbildungsmethoden der Darstellenden Geometrie. Sitz.-Ber. Akad. Wiss. Wien (math.-naturw. Kl.) 132: 177-192

[8] ECKHART, L. (1923) Konstruktive Abbildungsverfahren. Wien

[9] GIERING, O. (1982) Vorlesungen über höhere Geometrie. Vieweg, Braunschweig

[10] GRÜNWALD, J. (1911) Ein Abbildungsprinzip, welches die ebene Geometrie und Kinematik mit der räumlichen Geometrie verknüpft. Sitz.-Ber. Akad. Wiss. Wien 12: 677-741

[11] HAVLICEK, H. (1983) Die linearen Geradenabbildungen aus drei-dimensionalen projektiven PAPPOS-Räumen. Sitz.-Ber. Akad. Wiss. Wien (math.-naturw. Kl.) 192: 99-111

[12] HoscheK, J. (1971) Liniengeometrie. Bibliographisches Institut, Zürich

[13] JÜTTLER, B. (1993) Zur Konstruktion rationaler Kurven und Flächen auf Quadriken. J. Geom. 47: 53-64

[14] LüBberT, CH. (1977) Über eine Klasse von linearen Geradenabbildungen. Preprint No. 341, TH Darmstadt

[15] MüLLER, H. R. (1962) Sphärische Kinematik. VEB Deutscher Verlag der Wissenschaften, Berlin

[16] Peternell, M., Pottmann, H. (2000) Interpolating Functions on Lines in 3-Space. In: Curves and Surface Fitting, St. Malo, 1999 (COHEN, A., RABUT, CH., SCHUMAKER, L. L., eds.), pp. 351-358. Vanderbilt Univ. Press, Nashville, TN 
[17] Pottmann, H., Wallner, J. (2001) Computational Line Geometry. Springer, Berlin

[18] ReHвоск, F. (1926) Die linearen Punkt-, Ebenen- und Strahlabbildungen der Darstellenden Geometrie. Zeitschr. angew. Math. u. Mech. 6: 379-400, 449-468

[19] ReHbock, F. (1931) Zur Abbildung des Punkt- und Ebenenraumes auf die Kinematik der hyperbolischen und elliptischen Ebene. Mh. Math. u. Phys. 38: 257-274

[20] STUDY, E. (1903) Geometrie der Dynamen. Teubner, Leipzig

[21] WeISS, E. A. (1937) Einführung in die Liniengeometrie und Kinematik. Teubner, Leipzig

[22] Wunderlich, W. (1960) Eckhart-Rehbocksche Abbildung und Studysches Übertragungsprinzip. Publ. Math. Debrecen 7: 94-107

Anschrift des Verfassers: Mag. Dr. Boris Odehnal, Institut für Diskrete Mathematik und Geometrie, Technische Universität Wien, Wiedner Hauptstraße 8-10, 1040 Wien, Österreich, E-Mail: boris@geometrie.tuwien.ac.at. 